\title{
A Novel Bandwidth Broker Architecture Based on Topology Aggregation in Delay|Bandwidth Sensitive Networks
}

\author{
Walid Htira ${ }^{1}$, Olivier Dugeon ${ }^{1}$, and Michel Diaz ${ }^{2}$ \\ 1 Orange Labs, \\ 2, avenue pierre marzin, 22300-France \\ \{walid.htira, olivier.dugeon @orange-ftgroup.com \\ ${ }^{2}$ Universite de Toulouse, \\ 7, avenue du Colonel Roche, 31077-France \\ michel.diaz@laas.fr
}

\begin{abstract}
Either in flow-based or class-based QoS architectures, controlling the admission of traffic entering the network becomes a crucial task in the new telecom services. The Bandwidth Broker BB architecture is one of the efficient admission control solutions. In this paper, we present a novel bandwidth broker architecture for scalable support of guaranteed services based on the concept of topology aggregation. Indeed, the bandwidth broker does not manage all the detailed information, about topology and reservation, stored in the admission control database. Instead, it uses an aggregated representation in order to decide if a new service request can be accepted or rejected. We demonstrate that the reduction of the amount of information managed by the bandwidth broker enhance the performance of the admission control function thereby increasing the overall call processing capability.
\end{abstract}

Keywords: Admission control, bandwidth broker, topology aggregation, service guarantee.

\section{Introduction}

Ensuring minimum quality-of-service (QoS) levels to the new emerging telecom services is an important challenge for future packet networks. Toward this end, a number of Call Admission Control (CAC) approaches have been drawn to reserve network resources in order to ensure that users and QoS objectives can be satisfied. The Resource reservation protocol RSVP [1] is one of these approaches. It consists in establishing and maintaining the resource reservation in an IntServ 2. network. Although this signalling protocol is very strong in providing QoS support, it is not scalable, since it is necessary to maintain a flow state in each router along the flow's path. Contrary to RSVP, in the DPS (Dynamic Packet State) technique [3], the flow state information is inserted into packet headers, which overcomes the need for per-flow signaling and state management. The key problem encountered with this approach is the necessity that all routers 
in the flows path participate in the admission control process, implement the same scheduling mechanism and update packet headers. Trying to reduce the overhead generated by the two aforementioned approaches, other measurement based solutions have been proposed: The egress admission control [4] which is based on passive monitoring of the network. Although this mechanism provides an efficient service model and scalability, it requires specific functionalities in the core and edge routers, like insertion of packet state in the headers, special scheduler and rate monitoring. To prevent the use of a signaling protocol and special packet processing in core nodes, a call admission control mechanism based on probing [5] was proposed, where a test flow is inserted into the network to measure its congestion level.

Instead of having a distributed admission control, a Bandwidth Broker (BB) was proposed [6] within the DiffServ [7] model in order to concentrate in only one element the admission control functions. This entity maintains an admission control database with information about network topology and QoS state of each path and node. It also maintains a routing table allowing it to determine the path that the admitted flow will traverse towards the receiver. So, when a new flow with specific traffic parameters requests admission, the bandwidth broker calculates the bandwidth availability in each link of the path followed by this new flow to decide if it can be admitted or not. If the flow is admitted, the $\mathrm{BB}$ sends a message to the sender with a positive answer to the flow's request, and updates its database. Thus, using this approach, the call admission control process is simplified. There is no need to store the QoS reservation states in the core routers. In addition, the BB offers a global vision of the network utilization which allows it to perform admission control function with much guarantee. However, a key difficulty encountered with such approach is the large amount of information to manage which leads to scalability issues in large networks. So, the more detailed the network is, the more time the admission control algorithm needs to compute the admission decision. There are two major solutions to keep this amount of information in a reasonable volume. The first one is to reduce the size of the network by splitting it into PoPs or areas. The major problem with this solution is how to conceive the coordination between BBs in order to not introduce much overhead in the network. The second solution is based on information aggregation. In this case, the main objective is to sufficiently aggregate the information to reduce the amount of data significantly without loosing too much detail which could lead the admission control algorithm to erroneous decisions.

In this paper, we design a novel model of bandwidth broker based on topology aggregation. Admission control decisions are made based on an abstract structure of the network topology formed only by border routers. There is no need to store information about the core routers in the topology database. Our goal is to develop an architecture for admission control that can simultaneously achieve scalability and a strong service guarantee without sacrificing network utilization. The detailed information about the interactions between the CAC server and the network domain routers are described in [8]. 
The remainder of the paper is organized as follows. Section 2 investigates the most important aggregation models presented in the literature. Then, we propose in the Section 3 our topology aggregation based BB model. Simulation results are analyzed in Section 4 and the conclusion follows in Section 5.

\section{Topology Aggregation Overview and Network Model}

This section starts by introducing the topology aggregation concept and then exposes the network model.

\subsection{Topology Aggregation Overview}

The topology aggregation concept consists in summarizing the topology information of a network domain into a more compact structure. Numerous solutions were proposed in the literature [9, [10, 11] [12, 13, the most important ones being; The full-mesh and the star schemes. The first one [14] uses a logical path between each pair of border nodes to construct a full-mesh aggregated topology. This representation is by far the most accurate one. Unfortunately, the amount of information to be advertised increases as the square of the number of borders. The second scheme 14], which is the star, consists in connecting border nodes via virtual paths to a virtual center called "nucleus". In order to minimize distortion, it is recommended to define directed paths between borders called "bypasses". The number of logical paths in the star representation should not exceed three times the number of border nodes B. The complexity of this approach is in the order of $B^{2}$. The performance of these two schemes was compared in [15]. It appears that the star is more powerful than the full-mesh where it significantly reduces the amount of topology information stored in the database.

\subsection{Network Model}

A large network is a set of domains and links that connect these domains. It is modeled as a directed graph $\mathrm{G}(\mathrm{V}, \mathrm{E})$, where $\mathrm{V}$ is the set of nodes and $\mathrm{E}$ is the set of directed links among the nodes in $\mathrm{V}$. In one domain, $\mathrm{B}$ is the set of border nodes that have links connected to nodes in other domains. The links in E are marked by a combination of additive and concave parameters. These parameters represent the level of QoS provided by each edge. Given a directed path $\mathrm{P}=(\mathrm{a}$, $\mathrm{b}, \mathrm{c}, \ldots, \mathrm{x}, \mathrm{y})$ with $(\mathrm{a}, \mathrm{b}, \mathrm{c}, \ldots, \mathrm{x}, \mathrm{y}) \in \mathrm{V}$, the value of these metrics over this path can be one of the following compositions :

Definition 1. A metric $m$ t is additive if:

$m t(P)=m t(a, b)+m t(b, c)+\ldots+m t(x, y)$. Delay, jitter and cost follow the additive composition rule.

Definition 2. A metric $m$ t is concave if: $m t(P)=\min (\operatorname{mt}(a, b), \operatorname{mt}(b, c), \ldots, m t(x, y))$. Bandwidth parameter follows the concave composition rule. 
In the rest of the paper, we study the case of delay|bandwidth sensitive networks. For this, we use $d l_{P}$ to denote the delay of the logical path corresponding to P. Referring to definition.1, it is equal to the sum of all delays through P. We use also $b w_{P}$ to denote the bandwidth of the logical path corresponding to P. Referring to definition.2, it is equal to the minimal bandwidth value through P.

\section{The Topology Aggregation Based Bandwidth Broker Architecture}

In order to resolve the scalability issue within the classical Bandwidth Broker design, this section presents a novel approach to aggregate the network topology in order to reduce the size of the call admission control database and then to enhance the performance of the bandwidth broker. We propose two star designs, one which is basic and another which is service guarantee oriented. In such designs, admission control is done on a per domain basis instead of on a hopby-hop basis. Thus, the complexity of the admission control algorithms may be significantly reduced. For illustration purposes, we consider a simple AS with nine routers and twelve bidirectional and symmetric links. Each link is identified by a (delay, bandwidth) pair. Four nodes are selected as borders, which are, R3, R5, R8 and R9 [Fig.1-a].

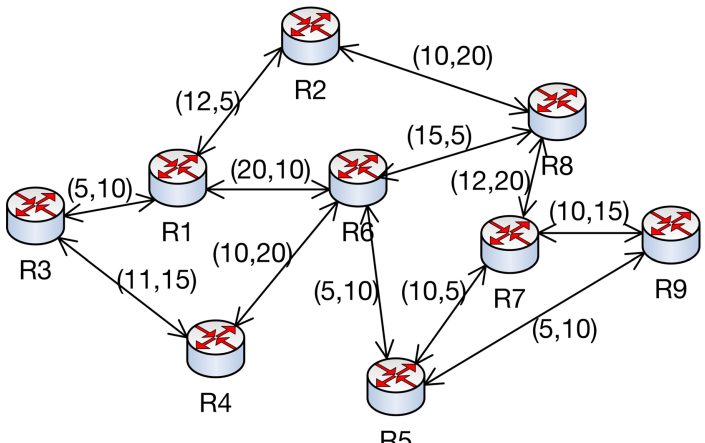

a) Network domain topology

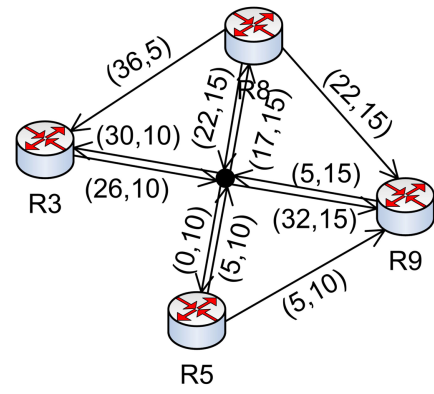

b) Star aggregated topology

Fig. 1. The star aggregation process

\subsection{The Basic Star Design}

As there is no common way or recommendation to retrieve the star representation, we propose new equations to compute spokes and bypasses.

The formation of the star begins by computing all the end to end network state information in the network domain. So, based on routing information, we compute logical paths between border routers referring to definition 1 and 2 . Indeed, the QoS metrics of a logical path correspond to the minimal bandwidth and the sum of delays through the physical path. For example, in Figure 1-a, to 
set a logical path between border routers R3 and R5, we compute the sum of delays and the minimal bandwidth through the route $R 3 \rightarrow R 4 \rightarrow R 6 \rightarrow R 5$, which gives a logical path with the metric $(26,10)$.

Once all the end to end logical paths are set, the star representation is established by splitting this logical paths into two spokes. The nucleus plays the role of a relay which interconnects all the border routers through spokes.

Our design follows three steps to find the QoS information associated to the basic star design: i) find the QoS parameters of the spokes from the border routers to the nucleus, ii) find the QoS parameters of the spokes from the nucleus to the border routers and iii) set the bypasses between border routers in order to reduce the distortion introduced by the aggregation process. The detailed information of the equations which lead to compute spokes and bypasses are described in [16].

In order to distinguish QoS parameters associated with end-to-end logical paths and spokes, we use respectively "t" and "s" notations. So, given a set of border routers $\mathrm{B}$, a nucleus $\mathrm{n}$, the metrics $M_{S n}^{s}$ and $M_{n D}^{s}$ denote respectively the QoS parameters of the ingoing spokes and the outgoing spokes.

$\forall S \in B$,

$$
M_{S n}^{s}=\left(\min \left(d l_{S D}^{t}\right), \max \left(b w_{S D}^{t}\right)\right), D \in B, D \neq S
$$

$\forall D \in B$,

$$
M_{n D}^{s}=\left(\max \left(d l_{S D}^{t}-d l_{S n}^{s}\right), \max \left(\min \left(b w_{S D}^{t}, b w_{S n}^{s}\right)\right)\right), S \in B, S \neq D
$$

For bypasses, we set B bypasses. The selection of these bypasses is made according to the comparison of the joint of the two spokes $S \rightarrow n, n \rightarrow D$ and the end-to-end logical path $\mathrm{S} \rightarrow \mathrm{D}$. When the deviation is large, we add a bypass between these two border routers. Figure 2-b shows the resulting basic star scheme. Applying admission control on this scheme shows that there is an overestimation of the network capabilities [see Section 4]. This results from the fact that the aggregated topology looks like a black box, so there is no manner to efficiently manage the spokes bandwidth. Figure 2 shows a typical example of a path aggregation which leads to a network capability over-estimation. A reservation of $5 \mathrm{Mb} / \mathrm{s}$ on the logical path XY exhausted all the available bandwidth on the physical link XW. While in the aggregated scheme, logical path XZ keeps $5 \mathrm{Mb} / \mathrm{s}$ free which is not true.

Even if the over-estimation value is not important, it can be the object of service degradation or user dissatisfaction. For this, we estimate that an acceptable
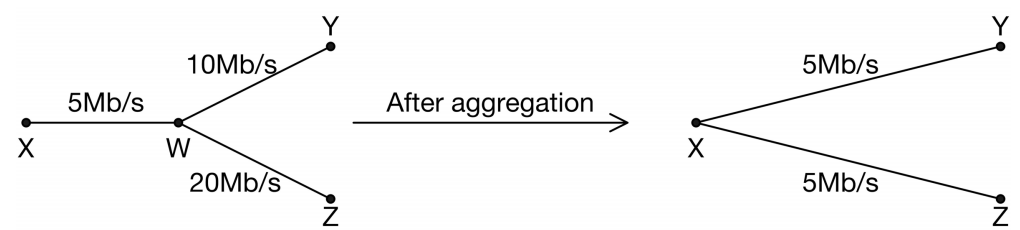

Fig. 2. Example of aggregation model accuracy limitation 
over-estimation percentage must not be greater than $0.5 \%$ of the overall number of connection requests treated during a time interval $\Delta t$. In the next subsection, we show a novel manner to form the star with much more service guarantee.

\subsection{The Guarantee-Based Star Design}

To be able to manage the bandwidth inside the aggregated topology, we have to fulfill all constraints coming from bandwidth utilization. Our analysis showed that it is needed to define on each spoke the physical link which can be an object of a maximal congestion in order to limit the over-estimation of network resources. But, as the basic star model is not defined directly from the real topology, it is not possible to find the set of physical links composing a spoke and so to infer the bottleneck link. So, our philosophy is to try to project the virtual nucleus in the real topology in order to seek the router which corresponds well to the nucleus characteristics, i.e is to solve the corresponding problem as follows i) to find in the real topology the real router that is the best potential nucleus node, ii) to drive from it the set of links and spokes, and iii) to use the resulting topology to perform the admission control for the given domain.

The logic of our approach is the following. For each core router we compute an approximate star scheme where the nucleus corresponds to the chosen core router and spokes corresponds to the partial routes which interconnect the core router to its borders. We use $H$ to denote the set of these partial routes. The QoS parameters of the partial routes are computed according to definition 1 and 2 and based on the routing protocol which indicates the route between the core router and the borders. At the end of this step, we are able to compute for each core router a nucleus deviation $n d$ which reflects the deviation between the basic star and the computed star schemes. Given a core router $\mathrm{cr}$, this deviation is computed from the sum of deflections from spokes between $\mathrm{cr}$ and border routers in the calculated star scheme and the spokes between the virtual nucleus and border routers in the basic star representation. Once the nucleus deviation is computed for each core router, we chose the node which has the minimal nucleus deviation to be the representative point of the virtual nucleus [Algorithm.1]. The deflection between two spokes is calculated as the Euclidean distance between two points in the delay|bandwidth plane due to the fact that spokes are represented as a (delay,bandwidth) pair.

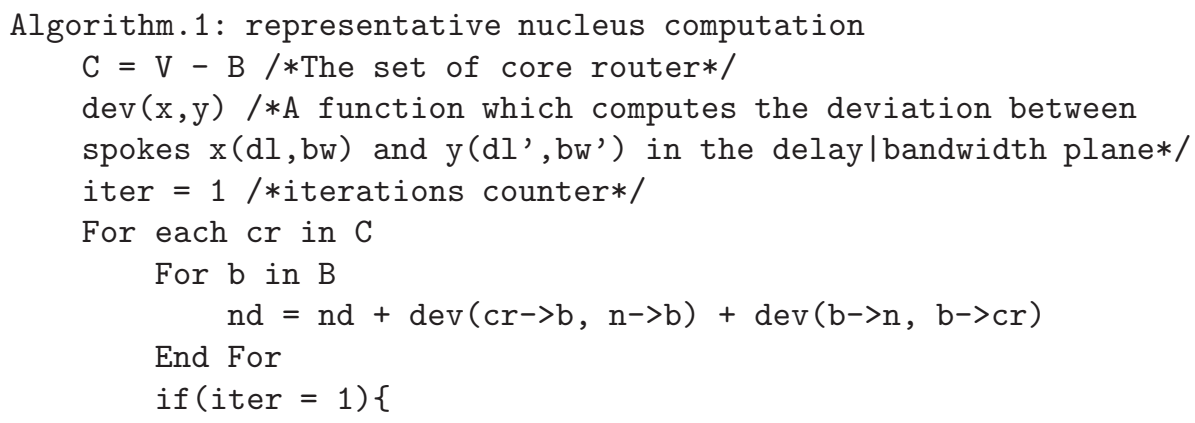




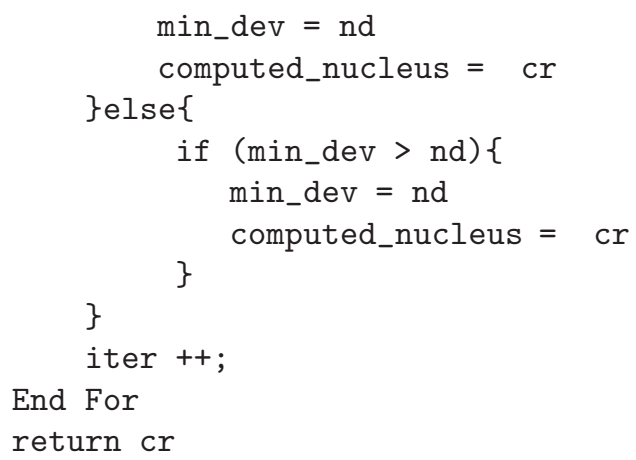

Now, once a router is selected to be the representative of the nucleus, it is possible to find each bottleneck link between the computed nucleus and border routers. This bottleneck link is defined as follows:

Definition 3. A bottleneck link BL of a partial route rt corresponds to the physical link having the minimal bandwidth value and crossed by the greatest number of partial routes. We denote $O_{r t}$ the set of partial routes crossing rt in the bottleneck link BL [Algorithm.2].

Algorithm.2: BL computation for a partial route rt

$\mathrm{L}(r \mathrm{t}) / *$ denotes the set of links in rt*/

Ml (rt) /*denotes the set of links belonging to L(rt)

and having the minimal bandwidth*/

For each link 1 in Ml(rt)

$N(1)=$ the number of partial routes crossing 1

$F(1)=($ bandwidth of link 1$) / N(1)$

End For

$\mathrm{BL}=$ link 1 which has the minimal $\mathrm{F}(1)$. end.

Thus, in the admission control process, it is necessary to check an additional condition in order to not over-estimate the network capabilities. This condition guarantees that the sum of reservation on all the partial route which cross the BL should be less or equal than the bandwidth capacity of the BL. So, given a network domain $\Omega$, the bandwidth sharing constraint to check for each partial route is the following : $\forall r t \in H$

$$
\sum_{r s v}\left(\sum_{r t^{\prime} \in O_{r t}} b w_{r t^{\prime}}\right)+\sum_{r s v} b w_{r t} \leq b w_{B L(r t)}
$$

Note that the BL is fixed for bypasses in the same manner that in spokes, but instead of using partial routes we use complete routes.

\subsection{Admission Control Process}

Suppose that the bandwidth broker receives a service request $\mathrm{Rq}$ from a router $\mathrm{S}$ to reach another router $\mathrm{D}$ in the same network domain. In our design, the 
$\mathrm{BB}$ has to verify if there is a direct bypass between these two routers in the database or not. If a bypass is found, three conditions have to be checked; $(4)(5)$ verify respectively the $\mathrm{Rq}$ admissibility in terms of bandwidth and delay, and (6) checks the bottleneck link constraint.

$$
\begin{gathered}
R q\left(b w_{S \rightarrow D}\right)+\sum_{r s v} b w_{S \rightarrow D} \leq b w_{S \rightarrow D} \\
R q\left(d l_{S \rightarrow D}\right)>d l_{S \rightarrow D}^{m} \\
R q\left(b w_{S \rightarrow D}\right)+\sum_{r s v} b w_{S \rightarrow D}+\sum_{r s v}\left(\sum_{r t^{\prime} \in O_{S \rightarrow D}} b w_{r t^{\prime}}\right) \leq b w_{B L(S \rightarrow D)}
\end{gathered}
$$

In the case where there is no bypass, five conditions have to be checked; $(7)(8)$ verify the availability of bandwidth in the two spokes, (9) checks the admissibility of Rq in terms of delay and (10)(11) verify the BL constraints on each spoke.

$$
\begin{gathered}
R q\left(b w_{S \rightarrow D}\right)+\sum_{r s v} b w_{S \rightarrow n} \leq b w_{S \rightarrow n} \\
R q\left(b w_{S \rightarrow D}\right)+\sum_{r s v} b w_{n \rightarrow D} \leq b w_{n \rightarrow D} \\
R q\left(d l_{S \rightarrow D}\right) \geq d l_{S \rightarrow n}+d l_{n \rightarrow D} \\
R q\left(b w_{S \rightarrow D}\right)+\sum_{r s v} b w_{S \rightarrow n}+\sum_{r s v}\left(\sum_{r t \in O_{S \rightarrow n}} b w_{r t}\right) \leq b w_{B L(S \rightarrow n)} \\
R q\left(b w_{S \rightarrow D}\right)+\sum_{r s v} b w_{n \rightarrow D}+\sum_{r s v}\left(\sum_{r t \in O_{n \rightarrow D}} b w_{r t}\right) \leq b w_{B L(n \rightarrow D)}
\end{gathered}
$$

Note that the admission control process is easier in the case of the basic star design because there is no need to check the BL constraints (6), (10) and (11).

This concept of topology aggregation based CAC can be extended to multiple Class of Services (CoSs): i) by splitting the delay|bandwidth aggregated topology proportionally to the bandwidth requirements for each $\mathrm{CoS}$, ii) by computing the delay|bandwidth aggregated scheme for each CoS (using the CoS allocated bandwidth instead of the physical bandwidth of the links).

\section{Simulation Results}

This section carries out some simulations to quantify the performance of our topology aggregation based bandwidth broker model compared to a traditional bandwidth broker architecture. 


\subsection{Network Model}

We use the topologies of four famous commercial backbones (AT\&T, Abovenet, Exodus and Sprintlink) for our experiments [17] 18]. Each experiment consists of two phases, the aggregated scheme formation and the admission control phase. In each backbone domain, the admission control phase consists in soliciting the bandwidth broker with 1.000 .000 connection requests generated uniformly by the border routers with bandwidth requirements of $128 \mathrm{~Kb}-5 \mathrm{Mb}$. A shortest path algorithm was used to determine the routes in the network domain.

Table 1. "Experimental topologies used for simulation"

\begin{tabular}{|l|c|c|c|c|}
\hline AS Name & Sprintlink & Exodus & Abovenet & AT\&T \\
\hline Backbone Routers & 465 & 211 & 244 & 729 \\
\hline AS border Routers & 56 & 32 & 39 & 46 \\
\hline
\end{tabular}

\subsection{Performance Measurement}

As mentioned earlier our experiments consist of two phases, namely the aggregated scheme formation phase and the admission control phase. For the first phase, simulations show that the basic star design needs less time to be computed than the guarantee based star. This is normal because there is no need to retrieve the representative nucleus and further to compute the corresponding BLs. The aggregation time $\tau$ of the basic star as well as the guarantee based star model is in the order of seconds [Table.2]. We can also remark, that $\tau$ is in the order of a routing protocol time convergence like OSPF. So, even in case of re-routing, the reformation time of the star is still acceptable and do not delay the admission control process [see Table.2].

Table 2. "Evaluation of the star formation time within the two star designs"

\begin{tabular}{|c|l|c|c|c|c|}
\hline Eval parameter & BB design & Sprintlink & Exodus & Abovenet & AT\&T \\
\hline \multirow{2}{*}{$\tau$ (seconds) } & Basic Star & 52 & 6 & 11 & 36 \\
& Guarantee-based star & 73 & 9 & 16 & 70 \\
\hline
\end{tabular}

The second phase, which concerns the admission control process, shows the strength of our design in reducing the amount of information stored in the database and so enhancing the admission control decision quickness. Compared to a traditional bandwidth broker model, our architecture reduces significantly the size of the database by using a simple summarized topology structure. So, admission control decisions, resources reservation and resources release are made in two passes by checking spokes state or in one pass by checking the bypass resources availability. Simulation results show that the database of the guarantee based star is slightly bigger than that of the basic star. This results from the fact that the guarantee-based model stores besides the end-to-end topology representation, the BLs information. Table. 3 shows the percentage of our BB database 
size $\beta$ by comparison with the total size needed by the traditional BB model to store the information about routing, reservations and topology. Despite that the guarantee based star design database is slightly bigger than that of the basic star model, our two designs outperform greatly the traditional bandwidth broker architecture. The database size is at least 43 times smaller in the case of the basic star design and 35 times smaller in the case of the guarantee-based star design. A such remarkable reduction has permit the enhancement of the admission decision time. There is no need to explore all the database and routing table in order to find the path followed by the incoming flow. Only an end to end check is needed to decide if the incoming call can be accepted or rejected.

The admission control decision time $\gamma$ is computed referring to the average time taken by the BB per new connection in order to make decision. Compared to the traditional BB architecture, $\gamma$ in our two designs is at least 3 times faster [see Table.3]. This Experiments show also that the decision time in the guarantee based star model is slightly larger than that of the basic star model, the difference is in the order of tens of microseconds $(\mu s)$. This results from the fact that there is no additional BL conditions to check. This quickness in making admission control decisions allows our two models to increase theirs call processing capability and so enhancing the BB availability.

Table 3. "The admission control performance evaluation"

\begin{tabular}{|c|l|c|c|c|c|}
\hline Eval parameter & BB design & Sprintlink & Exodus & Abovenet & AT\&T \\
\hline \multirow{2}{*}{$\beta$} & Basic star & $1.70 \%$ & $2.29 \%$ & $2.19 \%$ & $1.68 \%$ \\
& Guarantee-based star & $2.19 \%$ & $2.85 \%$ & $2.79 \%$ & $2.28 \%$ \\
\hline \multirow{3}{*}{$\gamma(m s)$} & Traditional BB & 3.393 & 2.664 & 2.660 & 3.012 \\
& Basic star & 0.390 & 0.319 & 0.609 & 0.465 \\
& Guarantee-based star & 0.420 & 0.450 & 0.700 & 0.523 \\
\hline
\end{tabular}

These two evaluation parameters prove the strength of our aggregated bandwidth broker architecture compared to the traditional design in term of complexity reduction, but do not prove its efficiency and its accuracy by comparison with the real network topology parameters. For this, we have conducted some experiments to evaluate the pertinence of our BB both when it is based on a basic star or on a guarantee-based star. For this, we introduce the exactness indicator $\varepsilon$ which presents the accuracy of our approach to accept or reject new request in accordance with the real network capabilities. This indicator denotes the percentage of right decisions taken by the aggregated design. $\varepsilon$ was computed for the two star designs. It appears that the guarantee-based star model is more accurate than the basic star as the number of right admission control decisions is higher. In the two star designs, $\varepsilon$ is always higher than $88 \%$.

By focusing on the erroneous decisions taken by the bandwidth broker, we wanted to analyze the over-estimation introduced by the aggregation process. For this, we defined the over-estimation percentage $\sigma$ which represents the percentage of connection requests admitted by excess whereas the network domain 
capabilities does not allow it. Simulation results show the utility of defining BLs in the reduction of the over-estimation percentage. Compared to the basic star design, it appears that the guarantee-based star is at least 9 times more accurate. It has a $\sigma$ at the most equal to $0.1238 \%$, which is a very good guarantee performance result that responds to our QoS suggestions [Table.4].

Table 4. "The topology based BB accuracy evaluation"

\begin{tabular}{|c|l|c|c|c|c|}
\hline Eval parameter & BB design & Sprintlink & Exodus & Abovenet & AT\&T \\
\hline \multirow{2}{*}{$\varepsilon$} & Basic star & $88.3905 \%$ & $96.4774 \%$ & $93.8795 \%$ & $92.2024 \%$ \\
& Guarantee-based star & $88.5064 \%$ & $96.4998 \%$ & $94.2668 \%$ & $92.5165 \%$ \\
\hline \multirow{2}{*}{$\sigma$} & Basic star & $0.7031 \%$ & $0.4001 \%$ & $0.6423 \%$ & $1.1834 \%$ \\
& Guarantee-based star & $0.0486 \%$ & $0.0442 \%$ & $0.0287 \%$ & $0.1238 \%$ \\
\hline
\end{tabular}

The aforementioned simulation results lead to several conclusions; i) Our bandwidth broker architecture, either it is basic or guarantee-based, outperforms largely the traditional BB design in terms of admission control algorithm complexity reduction, ii) The guarantee-based star design achieves very good results in terms of accuracy and overestimation percentage reduction. It presents a good track to investigate by Telecom operators for guaranteed and fast services delivery, iii) The basic star design offers very good scalability performance with a slight service degradation.

\section{Conclusion}

A broad view over the admission control approaches exhibits a tendency in adopting solution based bandwidth broker thanks to the service guarantee which it offers. This paper presents a novel bandwidth broker architecture for scalable support of guaranteed services based on the concept of topology aggregation. More specifically, the BB does not check all the detailed topology and reservation information. Instead, it uses a summarized picture of the network capabilities in order to perform admission control. Some simulations were conducted with four famous commercial backbone topologies. The experiments show that our architecture, either it is guarantee based or basic, reduces significantly the size of the admission control database as well as the admission control decision time. Compared to the basic star design, the guarantee-based model achieves very good performance in terms of accuracy and resources over-estimation reduction.

As an ongoing work, our future plan will focus on how to retrieve the representative nucleus without handling all the core routers in order to decrease even more the time of the guarantee-based star formation.

\section{Acknowledgment}

This work at Orange Labs, is registered under French patent $n^{\circ} 0707043$. 


\section{References}

1. Zhang, L., et al.: A New Resource ReSerVation Protocol. IEEE Network 7, 8-18 (1993)

2. Braden, R., et al.: Integrated Services in the Internet Architecture. Internet RFC 1633 (1994)

3. Stoica, I., Zhang, H.: Providing Guaranteed Services without per Flow Management. In: Proceedings of ACM SIGCOMM 1999, Cambridge, MA (August 1999)

4. Centinkaya, C., Knightly, E.: Scalable Services via Egress Admission Control. In: Proceedings of IEEE INFOCOM 2000, Tel Aviv, Israel (March 2000)

5. Qiu, J., Knightly, E.: Inter-class Resource Sharing using Statistical Service Envelopes. In: Proceedings of IEEE INFOCOM 1999, New York, NY (March 1999)

6. Zhang, Z., et al.: Decoupling QoS Control from Core Routers: a Novel Bandwidth Broker Architecture for Scalable Support of Guaranteed Services. In: Proceedings of ACM SIGCOMM 2000, Stockholm, Sweden (August 2000)

7. Blake, S., et al.: An Architecture for Differentiated Services. Internet RFC 2475 (1998)

8. Htira, W., Dugeon, O., Diaz, M.: STAMP: Towards A Scalable Topology Announcement and Management Protocol. In: IEEE AINA 2008, GinoWan, Okinawa, Japan (March 2008)

9. Awaebuch, B., Shavitt, Y.: Topology Aggregation for Directed Graphs. In: ISCC 1998, Athens, Greece (June 1998)

10. Iwata, A., Suzuki, H., Izmailow, R., Sengupta, B.: QoS aggregation algorithms In hierarchical ATM networks. In: ICC 1998 (June 1998)

11. Awerbuch, B., Du, Y., Khan, B., shavitt, Y.: Routing Through teranode networks with topology aggregation. In: ISCC 1998, Athens, Greece (June 1998)

12. Lee, W.C.: Spanning Tree Method for Link State Aggregation in Large Communication Networks. In: INFOCOM 1995, Boston, MA, USA (April 1995)

13. Lee, W.: Minimum Equivalent Subspanner Algorithms for Topology Aggregation in ATM Networks, Colmar, France (June 1999)

14. Bhutani, K.R., Battou, A., Khan, B.: Two Approaches for Aggregation of Peer Group Topology in Hierarchical PNNI Networks. In: The Catholic Uni. of America. Dept. of Mathematics, Washington, DC (June 1998)

15. Guo, L., Matta, I.: On State Aggregation for Scalable QoS Routing. In: ATM Workshop 1998 (May 1998)

16. Htira, W., Dugeon, O., Diaz, M.: An Aggregated Delay|Bandwidth Star Scheme For Admission Control. In: IEEE CAMAD 2007, Athens, Greece (September 2007)

17. CAIDA. Mapnet raw source data files, http://www.caida.org/tools/visualization/mapnet/Data/

18. Liu, J., Liljenstam, M., Nicol, D.: An internet topology for simulation, http://www.crhc.uiuc.edu/jasonliu/projects/topo/ 